\title{
Frequency Domain Approach to Fault Diagnosis of Analog Filters
}

\author{
Dražen Jurišić, Neven Mijat and Vladimir Čosić \\ Faculty of electrical engineering and computing Zagreb \\ Unska 3, Zagreb, 10000, Croatia \\ Fax (+385 1) 6129652 Phone (+385 1) 6129911 E-mail: drazen.jurisic@fer.hr
}

\begin{abstract}
This paper presents fault location technique for testing analog filters using a fault dictionary. The single hard faults of the filter passive elements can be located and identified. To enable the efficient testing an initial fault dictionary is optimised in order to get the minimal size of the dictionary with maximum number of uniquely recognised faults. After a simulation, the procedure tests the circuit and locates the failed element using adequate fault isolation criterion. The performances of the described method are illustrated with an analog filter fault diagnosis example performed in the frequency domain. The fault detection problem has also been considered.
\end{abstract}

\section{INTRODUCTION}

The component values in electrical circuits deviate from its nominals due to aging, temperature, etc. If a component value deteriorate outside its tolerance bounds producing a circuit failure, the fault isolation procedure can be carried out to locate the faulty element.

The described procedure includes linear and nonlinear circuits and can be applied in time as well as in the frequency domain. The necessary responses for nominal conditions and for a set of predetermined faults could be obtained using any general purpose circuit simulator. The presented method is illustrated by the frequency domain analog filter fault diagnosis [3].

The filter function magnitudes correspondent to particular single faults are calculated at a previously determined set of discrete test frequencies, forming a fault dictionary. The dictionary is stored in the test equipment, consisting of the Network analyser HP4195A connected via IEEE-488 interface bus to the computer and the appropriate software, including circuit simulator [4].

The magnitudes of the failed filter are compared on a least square criterion to those in the fault dictionary to determine which fault is occurred [4].

\section{FAULT DIAGNOSIS OF ANALOG CIRCUITS}

There exists a number of various techniques for analog circuits testing [1], convenient for testing specific types of circuits. Since no universal method exists, further improvements are possible.

The basic concept of the procedure was given in the preliminary paper [4]. The presented method in this communication includes proposal for fault detection using Monte Carlo simulation, definition of scale of marks, optimization of measurements and the isolation criterion. It is dedicated for testing analog filters, passive as well as active.

\subsection{Categorization of faults}

By the fault we mean any change in the element value with respect to its nominal value which can cause the failure of the circuit. The faults could be catastrophic (hard) if the faulty element produces either a short or an open circuit. For the changes outside tolerance bounds but without reaching the extreme values, the faults are called soft.

Simultaneous changes in several parameters can occur producing multiple fault. The most fault location techniques are concerned with single faults, when only one component is defective.

Two main problems arise in circuit testing: fault detection and fault location. To locate (or identify) the faulty component the appearance of the circuit fault has to be detected, first.

\subsection{Fault detection}

To propose a fault detection procedure, some standard methods such as worst case simulation or Monte Carlo statistical analysis can be used.

Let the network function be

$$
F\left(\mathbf{r}_{\mathbf{0}}, \tau, \omega\right) \text {, }
$$

where $\mathbf{r}_{0}$ and $\tau$ are $n$-dimensional vectors of nominal parameters and their tolerances and $\omega$ is angular frequency.

For small element changes $\Delta r_{i}$ the largest network function deviation (in the worst case sense) due to all element tolerances is

$$
\Delta F_{\mathrm{MAX}}(\omega)=\sum_{i=1}^{n}\left|\frac{\partial F(\mathbf{r}, \omega)}{\partial r_{i}}\right|_{\mathbf{r}=\mathbf{r}_{0}} \cdot\left|\Delta r_{i}\right|_{\max },
$$

where $\left|\Delta r_{i}\right|_{\max }=r_{0 i} \tau_{i}$.

The basic idea of the fault detection procedure is to compare the filter magnitude with its fault bounds determined by the worst case or Monte Carlo analysis (Fig. 1(a)). To identify the tested circuit as faulty, its magnitude has to go outside its upper and lower bounds (curve 1 on Fig. 1(b)). This will happen only if the value of at least one parameter extends over its tolerance boundaries.

The fault detection procedure does not need to be accomplished in the entire continuous frequency interval $\left[\omega_{a}, \omega_{b}\right]$. The number $n_{f}$ of appro- 
priate discrete frequencies forming an $n_{f}$ dimensional vector $\omega$ can be chosen instead. The network function (1) becomes discrete

$$
F\left(\mathbf{r}_{\mathbf{0}}, \tau, \omega\right) \text {. }
$$

The circuit is free from faults when

$$
F\left(\mathbf{r}_{\mathbf{0}}, \omega_{i}\right)-\Delta F^{\mathrm{L}}{ }_{\operatorname{MAX}}\left(\omega_{i}\right) \leq F\left(\omega_{i}\right) \leq F\left(\mathbf{r}_{\mathbf{0}}, \omega_{i}\right)+\Delta F^{\mathrm{H}}{ }_{\text {MAX }}\left(\omega_{i}\right)
$$$$
\text { for } i=1, \ldots, n_{f} \text {. }
$$

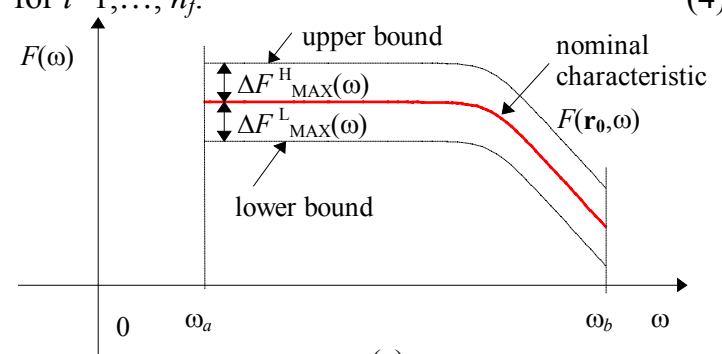

(a)

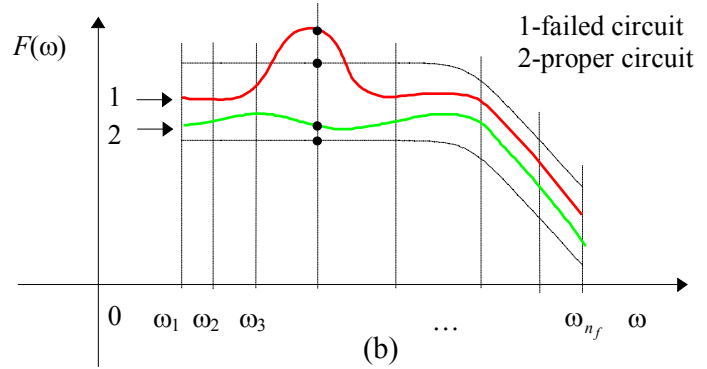

Fig. 1. Magnitude characteristic (a) nominal with worst case bounds, (b) failed and proper circuit.

For large component value deviations Monte Carlo technique should be used. It assumes that parameters $r_{i}$ are normally distributed (correlated or uncorrelated) random variables. According to the component values distributions a set of random parameter vectors $\mathbf{r}$ is generated. The magnitude of circuit's transfer function is determined for every sample.

The procedure is very simple but since a lot of calculations are needed it is very time consuming.

\subsection{Categorization of fault location and identification techniques}

The fault location procedures could be classified depending on a number of different techniques such as fault dictionary, parameter identification, fault verification, approximation, artificial intelligence techniques, etc. [1].

According to the stage in the testing process when the circuit is simulated the simulationbefore-test and the simulation-after-test approaches can be distinguished.

In the majority of cases, the fault dictionary technique belongs to the simulation-before-test approach.

The main steps in that technique are:

- fault definition

- the choice of response and domain

- selection of optimal set of measurements

- isolation of faults

\section{ANALOG FILTERS FAULT LOCATION USING FAULT DICTIONARY}

The technique is illustrated by an example of 4th order Butterworth low pass filter shown in the Fig. 2 [4], with the following specifications: attenuation in pass band $A_{p}=1 \mathrm{~dB}(0<f<20 \mathrm{~Hz})$, and in stop band $A_{s}=8 \mathrm{~dB}(30 \mathrm{~Hz}<f)$. The filter is realized by cascading two 2 nd order sections according to Fig. 2(a). Nominal element parameters are given in Table I (the values are in $[\Omega]$ and $[\mathrm{nF}]$ ).

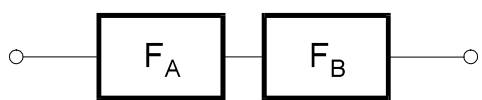

(a)

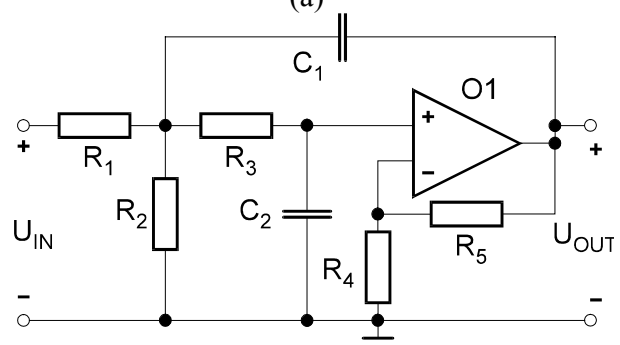

(b)

Fig. 2. Active 4th order LP filter (a) cascaded sections, (b) biquadratic section.

TABLE I Nominal parameters

\begin{tabular}{|c|ccccccc|}
\hline Block & $R_{1}$ & $R_{2 \bullet}$ & $R_{3 \bullet}$ & $R_{4 \bullet}$ & $R_{5} \bullet$ & $C_{1}$ & $C_{2}$ \\
\hline A & 31954 & 25883 & 14300 & 8100 & 10000 & 470 & 470 \\
\hline B & 16477 & 108230 & 14300 & 65685 & 10000 & 470 & 470 \\
\hline
\end{tabular}

\subsection{Fault definition}

One of the earliest technique for fault definition is the Seshu and Waxmann approach which varies the element values $\pm 50 \%$ from its nominals [3]. In the present case it is assumed that all passive elements (14 ones) could be faulty. In such approach the open and short circuits could be considered as element value changes $\pm 50 \%$. It is convenient to name such faults with for example $R_{1}+$ or $R_{1}$-. For the sake of simplicity the faults are usually numbered with 0 to $27\left(n_{\text {faul }}=28\right)$.

\subsection{Initial fault dictionary}

In the presented example the circuit simulator calculates the magnitude in 100 discrete logarithmically spaced frequencies ranging from $10^{1} \mathrm{rad} / \mathrm{s}$ to $10^{3} \mathrm{rad} / \mathrm{s}$, denoting them with numbers $0,1, \ldots, 99\left(n_{\text {freq }}=100\right)$.

Of course, it is too expensive to carry out procedures in all these frequency points. There are many ways to choose the subset of test frequencies for the initial fault dictionary $[1,3]$.

The $R_{1} \pm 50 \%$ deviation influence to the filter magnitude is illustrated in the Fig. 3. In the error characteristic there is a noticeable frequency point with the greatest gradient, indicating that in the 
neighbourhood of that frequency the influence of the fault is dominant. Similar influence holds for all other elements, yielding the initial set $\{47,50$, $52,53,54,57,58,60,62,66,67,71\}$ of $n_{\text {freq }}=12$ test frequencies.

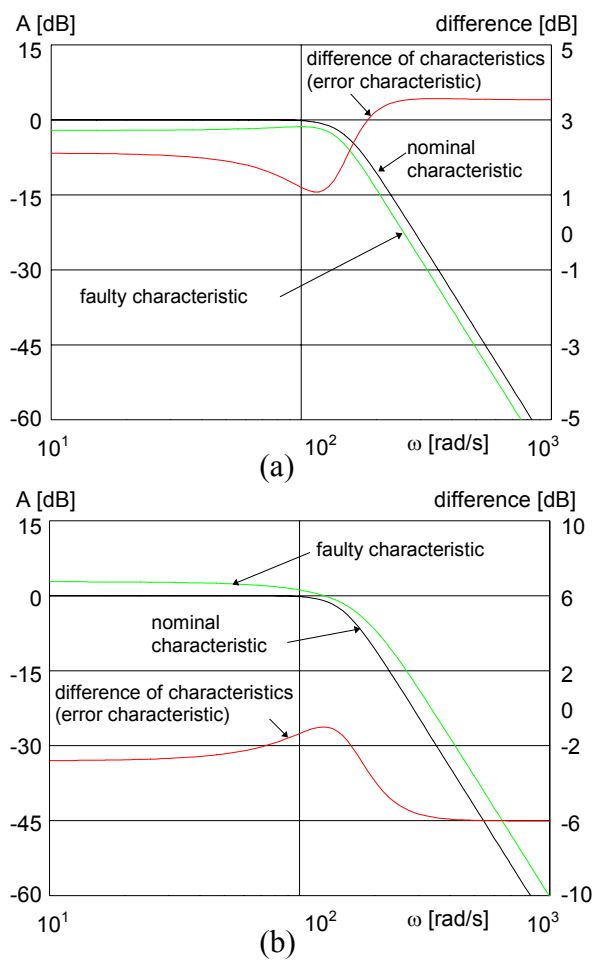

Fig. 3. Change of element's $R_{1}$ value (a) $+50 \%$, (b) $-50 \%$

TABLE II Initial Fault Dictionary

\begin{tabular}{|c|c|c|c|c|c|c|c|c|c|c|c|c|c|}
\hline \multicolumn{2}{|c|}{ Test Frequency } & \multicolumn{12}{|c|}{\begin{tabular}{|l|l|l|l|l|l|l|l|l|l|l|l|}
47 & 50 & 52 & 53 & 54 & 57 & 58 & 60 & 62 & 66 & 67 & 71 \\
\end{tabular}} \\
\hline $\mathrm{Nr}$. & Fault & \multicolumn{12}{|c|}{ Signature } \\
\hline 0 & $R_{1 \mathrm{~A}}+$ & 1 & & 1 & 1 & 1 & 1 & 12 & 22 & 2 & 2 & & 2 \\
\hline 1 & $R_{1 \mathrm{~A}^{-}}$ & 5 & 5 & 5 & 5 & 5 & 5 & 56 & 66 & 6 & 7 & & 7 \\
\hline 2 & $R_{2 \mathrm{~A}^{+}}$ & 6 & 6 & 6 & 6 & 6 & 6 & & 55 & 5 & 0 & & 0 \\
\hline 3 & $R_{2 \mathrm{~A}^{-}}$ & 3 & & 3 & 3 & 3 & 3 & & 32 & 2 & 2 & & 1 \\
\hline 4 & $R_{3 \mathrm{~A}^{+}}$ & 1 & & 2 & 2 & 2 & 3 & 33 & 33 & 3 & 3 & & 3 \\
\hline 5 & $R_{3 \mathrm{~A}^{-}}$ & 1 & 0 & 0 & 0 & 0 & 5 & 67 & 78 & 8 & 8 & & 8 \\
\hline 6 & $R_{4 \mathrm{~A}^{+}}$ & 2 & 3 & 3 & 3 & 3 & 3 & 33 & 33 & 3 & 3 & & 2 \\
\hline 7 & $R_{4 \mathrm{~A}^{-}}$ & 7 & 7 & 7 & 7 & 7 & 8 & 88 & 87 & 7 & 7 & & 7 \\
\hline 8 & $R_{5 \mathrm{~A}}+$ & 6 & 7 & 7 & 8 & 8 & 8 & 88 & 88 & 8 & 7 & & 6 \\
\hline 9 & $R_{5 \mathrm{~A}^{-}}$ & 3 & 3 & 3 & 4 & 4 & 4 & 44 & 44 & 4 & 3 & & 3 \\
\hline 10 & $R_{1 \mathrm{~B}}+$ & 1 & 1 & 1 & 1 & 1 & 2 & 22 & 22 & 2 & 2 & & 2 \\
\hline 11 & $R_{1 \mathrm{~B}^{-}}$ & 5 & 5 & 5 & 5 & 5 & 6 & 66 & 66 & 6 & 6 & & 7 \\
\hline 12 & $R_{2 \mathrm{~B}}{ }^{+}$ & 0 & 0 & 0 & 0 & 0 & 0 & $0 \quad$ & $\begin{array}{ll}0 & 0\end{array}$ & 0 & 0 & & 0 \\
\hline 13 & $R_{2 \mathrm{~B}^{-}}$ & 1 & & 1 & 1 & 1 & 1 & 11 & 11 & 1 & 0 & & 0 \\
\hline 14 & $R_{3 \mathrm{~B}}+$ & 1 & & 1 & 1 & 1 & 2 & 22 & 22 & 2 & 2 & & 2 \\
\hline 15 & $R_{3 \mathrm{~B}^{-}}$ & 5 & 5 & 5 & 5 & 5 & 6 & 66 & 66 & 6 & 6 & & 7 \\
\hline 16 & $R_{4 \mathrm{~B}^{+}}$ & 1 & 1 & 1 & 1 & 1 & 1 & 1 & 11 & 1 & 1 & & 1 \\
\hline 17 & $R_{4 \mathrm{~B}^{-}}$ & 5 & 5 & 5 & 5 & 5 & 5 & 55 & 55 & 5 & 5 & & 5 \\
\hline 18 & $R_{5 \mathrm{~B}}+$ & 5 & 5 & 5 & 5 & 5 & 5 & 55 & 55 & 5 & 5 & & 5 \\
\hline 19 & $R_{5 \mathrm{~B}^{-}}$ & 1 & 1 & 1 & 1 & 1 & 1 & 11 & 11 & 1 & 1 & 1 & 1 \\
\hline 20 & $C_{1 \mathrm{~A}^{+}}$ & 7 & 8 & 8 & 8 & 8 & 7 & 60 & $\begin{array}{ll}0 & 1\end{array}$ & 1 & 2 & & 2 \\
\hline 21 & $C_{1 \mathrm{~A}^{-}}$ & 2 & 3 & 3 & 3 & 3 & 3 & 33 & 33 & 3 & 2 & & 0 \\
\hline 22 & $C_{2 \mathrm{~A}^{+}}$ & 2 & 3 & 3 & 3 & 3 & 4 & 4 & 44 & 4 & 3 & & 3 \\
\hline 23 & $C_{2 \mathrm{~A}^{-}}$ & 0 & 0 & 0 & 0 & 0 & 5 & 67 & 78 & 8 & 8 & & 8 \\
\hline 24 & $C_{1 \mathrm{~B}}+$ & 5 & 5 & 5 & 5 & 5 & 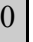 & $0 \quad$ & $\begin{array}{ll}0 & 1\end{array}$ & 1 & 1 & & 1 \\
\hline 25 & $C_{1 \mathrm{~B}^{-}}$ & 1 & 1 & 1 & 1 & 1 & 1 & 1 & $0 \quad 0$ & 0 & 0 & j & 5 \\
\hline 26 & $C_{2 \mathrm{~B}^{+}}$ & 2 & 2 & 2 & 2 & 2 & 2 & 2 & 2 & 3 & 3 & & 3 \\
\hline 27 & $C_{2 \mathrm{~B}^{-}}$ & 6 & 6 & 6 & 6 & 7 & 7 & 77 & 77 & 7 & & 8 & 8 \\
\hline
\end{tabular}

The records in the fault dictionary can be magnitudes or any other signature. There are $n_{\text {freq }} \times n_{\text {faul }}$ records in the dictionary. Seshu and Waxmann proposed quantization of the error characteristic using the scale of marks [3]. The scale is presented on the Fig. 4 and is defined by a test engineer. The errors in interval $[-0.5,0.5] \mathrm{dB}$ is marked with 0 , in interval $[0.5,2] \mathrm{dB}$ with 1 , etc.

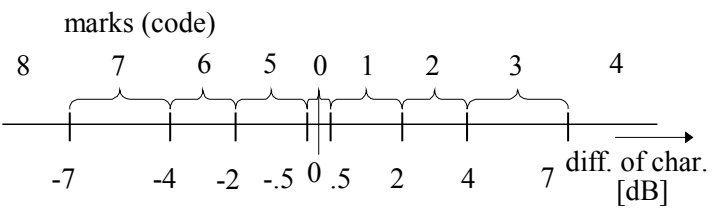

Fig. 4. Scale of marks associated with difference of characteristics.

The initial fault dictionary is presented on the Table II. Columns corresponds to test frequencies and rows corresponds to faults. Some of the faults

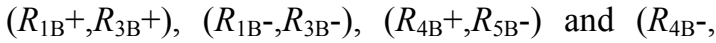
$R_{5 \mathrm{~B}}+$ ) have the same signatures, presenting the ambiguity. Other faults are uniquely defined.

\subsection{Optimizing the set of measurements}

In what follows the minimal number of test frequencies sufficient to isolate all uniquely identified faults should be determined (the shaded columns in Table II). To accomplish that the optimization method based on the modified binary logical manipulation similar to one proposed by Hochwald and Bastian is applied [2].

In the first step for each test frequency the faults are grouped concerning to the marks into ambiguity sets. These sets (i.e. the associated test frequencies) uniquely identify the faults applying the following rules:

- RULE-1 Any ambiguity set consisting of a single fault uniquely identifies that fault.

- RULE-2A Two ambiguity sets associated to two different test frequencies, whose intersection results in a single fault, uniquely identify that fault.

- RULE-2B Two ambiguity sets whose symmetric difference (the fault is the only different element and it is contained in only one set) results in a single fault, also uniquely define that fault. The RULE-2B can be extended on combinations of unions of ambiguity sets.

The optimization procedure makes the list with all single (RULE-1) and test frequency pairs (RULES-2A and 2B) uniquely defining the faults.

The procedure then searches throughout the list to find the minimal number of test frequencies which still isolate all of these unique faults. In our example there are two such a minimal combina- 
tions of three frequencies $(47,57,71$ and 47, 58, 71 ) and the former is shaded in Table II.

In the next stage the optimization procedure applies the same rules on the ununiquely defined faults with unshaded signatures in selected columns. The procedure groups the remaining ambiguous faults together.

Finally, optimized fault dictionary is constructed containing magnitudes instead of marks. There ununiquely isolated faults are enumerated on its end. The dictionary contains all necessities for testing, including frequency values in $[\mathrm{rad} / \mathrm{s}]$ and is presented in the Table III.

TABLE III Final Fault Dictionary

\begin{tabular}{|c|c|c|c|c|}
\hline \multicolumn{2}{|c|}{ Test Frequency } & $47(89 \mathrm{r} / \mathrm{s})$ & $57(142 \mathrm{r} / \mathrm{s})$ & $71(272 \mathrm{r} / \mathrm{s})$ \\
\hline Nr. & Fault & \multicolumn{3}{|c|}{ Magnitudes [dB] } \\
\hline 0 & $R_{1 \mathrm{~A}^{+}}$ & $-1.44 \mathrm{E}+00$ & $-3.86 \mathrm{E}+00$ & $-2.45 \mathrm{E}+01$ \\
\hline 1 & $R_{1 \mathrm{~A}^{-}}$ & $1.55 \mathrm{E}+00$ & $-1.02 \mathrm{E}+00$ & $-1.55 \mathrm{E}+01$ \\
\hline 2 & $R_{2 \mathrm{~A}^{+}}$ & $2.67 \mathrm{E}+00$ & $9.09 \mathrm{E}-02$ & $-2.10 \mathrm{E}+01$ \\
\hline 3 & $R_{2 \mathrm{~A}^{-}}$ & $-5.25 \mathrm{E}+00$ & $-7.92 \mathrm{E}+00$ & $-2.18 \mathrm{E}+01$ \\
\hline 4 & $R_{3 \mathrm{~A}^{+}}$ & $-1.10 \mathrm{E}+00$ & $-6.92 E+00$ & $-2.56 \mathrm{E}+01$ \\
\hline 5 & $R_{3 \mathrm{~A}^{-}}$ & $-5.81 \mathrm{E}-01$ & $-5.15 \mathrm{E}-01$ & $-1.06 \mathrm{E}+01$ \\
\hline 6 & $R_{4 \mathrm{~A}^{+}}$ & $-3.48 \mathrm{E}+00$ & $-7.72 E+00$ & $-2.41 \mathrm{E}+01$ \\
\hline 7 & $R_{4 \mathrm{~A}^{-}}$ & $4.78 \mathrm{E}+00$ & $5.71 \mathrm{E}+00$ & $-1.64 \mathrm{E}+01$ \\
\hline 8 & $R_{5 \mathrm{~A}^{+}}$ & $3.75 \mathrm{E}+00$ & $1.27 \mathrm{E}+01$ & $-1.76 \mathrm{E}+01$ \\
\hline 9 & $R_{5 \mathrm{~A}^{-}}$ & $-5.34 \mathrm{E}+00$ & $-1.02 \mathrm{E}+01$ & $-2.58 \mathrm{E}+01$ \\
\hline 10 & $R_{1 \mathrm{~B}}+$ & $-1.43 \mathrm{E}+00$ & $-4.36 \mathrm{E}+00$ & $-2.40 \mathrm{E}+01$ \\
\hline 11 & $R_{1 \mathrm{~B}^{-}}$ & $1.16 \mathrm{E}+00$ & $-1.24 \mathrm{E}-01$ & $-1.70 \mathrm{E}+01$ \\
\hline 12 & $R_{2 \mathrm{~B}}+$ & 2.37E-01 & $-2.03 \mathrm{E}+00$ & $-2.09 \mathrm{E}+01$ \\
\hline 13 & $R_{2 \mathrm{~B}^{-}}$ & $-9.54 \mathrm{E}-01$ & $-2.90 \mathrm{E}+00$ & $-2.13 \mathrm{E}+01$ \\
\hline 14 & $R_{3 \mathrm{~B}}+$ & $-1.34 \mathrm{E}+00$ & $-4.43 \mathrm{E}+00$ & $-2.41 \mathrm{E}+01$ \\
\hline 15 & $R_{3 \mathrm{~B}^{-}}$ & $8.42 \mathrm{E}-01$ & $-2.21 \mathrm{E}-01$ & $-1.68 \mathrm{E}+01$ \\
\hline 16 & $R_{4 \mathrm{~B}}+$ & $-6.40 \mathrm{E}-01$ & $-2.88 \mathrm{E}+00$ & $-2.15 \mathrm{E}+01$ \\
\hline 17 & $R_{4 \mathrm{~B}^{-}}$ & $1.55 \mathrm{E}+00$ & $-4.29 \mathrm{E}-01$ & $-1.94 \mathrm{E}+01$ \\
\hline 18 & $R_{5 \mathrm{~B}}^{+}$ & $7.55 \mathrm{E}-01$ & $-1.33 \mathrm{E}+00$ & $-2.02 \mathrm{E}+01$ \\
\hline 19 & $R_{5 \mathrm{~B}^{-}}$ & $-9.30 \mathrm{E}-01$ & $-3.19 \mathrm{E}+00$ & $-2.18 \mathrm{E}+01$ \\
\hline 20 & $C_{1 \mathrm{~A}^{+}}$ & $4.40 \mathrm{E}+00$ & $3.30 \mathrm{E}+00$ & $-2.44 \mathrm{E}+01$ \\
\hline 21 & $C_{1 \mathrm{~A}^{-}}$ & $-3.46 \mathrm{E}+00$ & $-8.01 \mathrm{E}+00$ & $-2.06 \mathrm{E}+01$ \\
\hline 22 & $C_{2 \mathrm{~A}^{+}}$ & $-3.37 \mathrm{E}+00$ & $-9.64 E+00$ & $-2.65 \mathrm{E}+01$ \\
\hline 23 & $C_{2 \mathrm{~A}^{-}}$ & $-5.46 \mathrm{E}-01$ & $-3.43 \mathrm{E}-01$ & $-1.03 \mathrm{E}+01$ \\
\hline 24 & $C_{1 \mathrm{~B}^{+}}$ & 7.98E-01 & $-2.07 \mathrm{E}+00$ & $-2.30 \mathrm{E}+01$ \\
\hline 25 & $C_{1 \mathrm{~B}^{-}}$ & $-9.46 \mathrm{E}-01$ & $-2.96 \mathrm{E}+00$ & $-1.98 \mathrm{E}+01$ \\
\hline 26 & $C_{2 \mathrm{~B}}+$ & $-2.88 \mathrm{E}+00$ & $-6.07 E+00$ & $-2.51 \mathrm{E}+01$ \\
\hline 27 & $C_{2 \mathrm{~B}^{-}}$ & $2.37 \mathrm{E}+00$ & $2.89 \mathrm{E}+00$ & $-1.33 \mathrm{E}+01$ \\
\hline
\end{tabular}

\subsection{Fault isolation}

At the time of testing, the magnitude of the failed analog filter is measured in the same test frequencies as in the dictionary in Table III. The fault isolation criterion is applied to the measured magnitudes and to the simulated magnitudes from the dictionary in order to locate the fault.

One fault isolation criterion has been proposed by Hochwald and Bastian [2], called "the nearest neighbour rule". This criterion picks up the fault case with the lowest value of $d$ (Euclid's distance)

$$
d=\sum_{i=1}^{n_{f o}}\left(H^{(j)}\left(\omega_{i}\right)-H\left(\omega_{i}\right)\right)^{2}
$$

where $H^{(j)}\left(\omega_{i}\right)$ is the $j$ th fault magnitude stored in dictionary, $H\left(\omega_{i}\right)$ is an actual filter magnitude and $n_{f o}$ is the optimized number of test frequencies.

For the filter from Fig. 2 the fault " $R_{5 \mathrm{~A}}$ in short circuit" was actually performed on a breadboard and the magnitudes were measured at three test frequencies.

The values of $d$ ranging from $d=2.1$ (fault $R_{5 \mathrm{~A}^{-}}$) to $d=369.3$ (fault $C_{2 \mathrm{~B}}+$ ) with the nearest value of $d=9.1$ (fault $C_{2 \mathrm{~A}}+$ ) were obtained correctly indicating a fault named " $R_{5 \mathrm{~A}}$-".

If one of ambiguous faults has been detected (e.g. $R_{1 \mathrm{~B}}+$ ), one should replace all elements in the corresponding ambiguous faults group $\left(R_{1 \mathrm{~B}}\right.$ and $R_{3 \mathrm{~B}}$ ) to fix up the circuit. Such faults which cannot be uniquely identified produce similar outputs and the isolation criterion may indicate the wrong component for replacement.

\section{CONCLUSIONS}

This paper presents fault location technique in analog networks particularly in analog filters. The technique is capable of locating single hard faults of filter passive elements such as short or open circuits. It belongs to the simulation-before-test approach and builds fault dictionary of magnitudes in frequency domain.

The efficiency of presented isolation method depends on the initial set of test frequencies and the predefined scale of marks. As the goal is to uniquely isolate all predefined faults with a minimal set of test frequencies, further improvements in the definition of the mark scale and initial test frequencies are desirable.

The optimizing the set of measurement method is very efficient. Since it handles only numbers, can be applied in all fault dictionary based techniques.

\section{REFERENCES}

[1] J. W. Bandler, A. E. Salama, "Fault diagnosis of analog circuits," in Proc. IEEE, Vol. 73, No. 8, pp. 1279-1325, 1985.

[2] W. Hochwald, J. D. Bastian, "A dc approach for analog fault dictionary determination," IEEE Trans. Circuits Syst., vol. CAS-26, pp. 523-529,1979.

[3] S. Seshu, R. Waxmann, "Fault isolation in conventional linear systems-A feasibility study," IEEE Trans. Reliab., vol. R-15, pp. 11-16, 1966.

[4] D. Jurišić, V. Čosić, N. Mijat, "Fault analysis of analog networks using fault dictionary," in Proc. 40th Anniversary Conference KoREMA, (Zagreb, Croatia), pp. 474-477, 1995, (in Croatian). 